\title{
MULTILOCUS SEQUENCE TYPING OF CARBAPENEM RESISTANT PSEUDOMONAS AERUGINOSA ISOLATES FROM PATIENTS PRESENTING AT PORT ELIZABETH HOSPITALS, SOUTH AFRICA
}

\author{
D. Annear ${ }^{1}$, J. Black ${ }^{2,3}$ and S. Govender ${ }^{1 *}$
}

${ }^{1}$ Department of Biochemistry and Microbiology, Nelson Mandela Metropolitan University, Port Elizabeth, South Africa. ${ }^{2}$ Department of Infectious Diseases, Livingstone Hospital, Port Elizabeth, South Africa. ${ }^{3}$ Department of Medicine, University of Cape Town, Cape Town, South Africa.

*Corresponding Author's E-mail: sharlene.govender@nmmu.ac.za

\begin{abstract}
Background: Pseudomonas aeruginosa is an important nosocomial pathogen that exhibits multiple drug resistance with increasing frequency, especially to carbapenems making patient treatment difficult. Carbapenem-resistance may be caused by porin gene mutations, active drug efflux, and carbapenemase production. This study evaluated the incidence of genes responsible for carbapenemase production in carbapenem-resistant Pseudomonas aeruginosa and assessed the genetic relatedness of the isolates by multi locus sequence typing (MLST).

Materials and Methods: Identification and antimicrobial susceptibility testing of $P$. aeruginosa isolates ( $\mathrm{n}=234)$ by the VITEK 2 system detected 81 carbapenem resistant $P$. aeruginosa isolates. PCR and DNA sequencing were used to screen isolates for three metallo- $\beta$-lactamase encoding genes. MLST included amplification of seven housekeeping genes and sequence type alignment using the online $P$. aeruginosa MLST database.

Results: Only the bla $a_{\mathrm{VIM}-2}$ gene was detected in 15 of the 81 carbapenem resistant isolates. MLST indicated six different novel sequence types among the bla $a_{\mathrm{VIM}-2}$ positive $P$. aeruginosa isolates with the majority of the isolates (9/15) containing identical allelic profiles of the sequence type allocated ST1 (provisionally assigned sequence type, awaiting addition of new sequence types to PubMLST database). Five of these ST1 isolates were from patients and an environmental sample in the same hospital ward suggesting an environmental reservoir. Carbapenem resistance in the bla $a_{\mathrm{VIM}-2}$ negative isolates may be due to other mechanisms.

Conclusion: The incidence of genes responsible for carbapenemase production in carbapenem-resistant Pseudomonas aeruginosa and genetic relatedness of these isolates in public healthcare facilities within the Port Elizabeth area is of concern and requires further investigation.
\end{abstract}

Keywords: Pseudomonas aeruginosa, VIM-2 carbapenemase, multi-locus sequence typing

\section{Introduction}

Pseudomonas aeruginosa is an important nosocomial pathogen that exhibits multiple drug resistance mechanisms with increasing frequency, especially to carbapenems making patient treatment difficult (Driscoll et al, 2007; Hirsch and Tam, 2010). Multidrug resistant $P$. aeruginosa infections are mainly treated with last line $\beta$-lactam antibiotics, carbapenems, (Rodríguez-Martínez et al, 2009) however the overuse and misuse of carbapenem antibiotics has contributed to the development of carbapenem resistance in $P$. aeruginosa by selectively promoting the growth and survival of $P$. aeruginosa strains containing carbapenem resistant genes (Queenan and Bush, 2007; Baumgart et al, 2010). Carbapenem-resistance may be caused by porin gene mutations, active efflux, and carbapenemase production (Rodríguez-Martínez et al, 2009; Nordmann et al, 2011). The mechanism depending on enzyme production is of greater importance due to the high level of resistance (Meletis et al, 2014). In addition, carbapenemases are encoded by genes located generally within mobile genetic elements, which facilitates their spread not only among strains, but also across species.

A variety of molecular methods have been used to type P. aeruginosa strains (Curran et al, 2004; Gomila et al, 2013), but these vary in their discriminatory potential. Multilocus sequence typing (MLST) is an alternative method for molecular typing which allows for comparison of the nucleotide sequence of a number of specific genes of different isolates and therefore allows for the characterization of a collection of clinical isolates (Curran et al, 2004; Belén et al, 2009).

Clinical $P$. aeruginosa isolates producing the VIM-2 carbapenemase have been reported in Cape Town and Port Elizabeth, South Africa (Jacobson et al, 2012; Govender et al, 2015). The objectives of this study were to evaluate the incidence of carbapenemase producing genes in carbapenem-resistant $P$. aeruginosa isolated from patients from public hospitals in Port Elizabeth, and to assess genetic relatedness of the isolates by MLST. 


\section{Materials and Methods Bacterial isolates}

P. aeruginosa isolates $(\mathrm{n}=234)$ were supplied by the National Health Laboratory Services (NHLS) from patients presenting at public hospitals in Port Elizabeth during March to August 2015. Identification and antimicrobial susceptibility profiles detected 81 carbapenem resistant $P$. aeruginosa isolates using the VITEK ${ }^{\circledR} 2$ instrument (bioMérieux) (control strain: P. aeruginosa ATCC 27853) and microbroth dilution respectively. Minimum inhibitory concentrations (MIC) were interpreted using the Clinical and Laboratory Standards Institute interpretive criteria (Clinical and Laboratory Standard Institute, 2015). Permission to conduct this study was obtained from the Department of Health and ethics approval granted from the NMMU Research Ethics Committee [Ref: H13-SCI-BCM-008].

\section{DNA Extraction}

Chromosomal and plasmid DNA were extracted from $200 \mu \mathrm{L} P$. aeruginosa culture using the DNeasy ${ }^{\circledR}$ blood and tissue and QIAprep ${ }^{\circledR}$ Spin Miniprep kits respectively, according to the manufacturer's (Qiagen) instructions. Extracted DNA was quantified using the NanoDrop ${ }^{\mathrm{TM}}$ Spectrophotometer (Thermo Scientific) at $260 \mathrm{~nm}$.

\section{Detection of carbapenemase encoding genes}

Primers and PCR conditions employed for detection of the metallo- $\beta$-lactamase encoding genes (bla $a_{\mathrm{VIM}}$, $b l a_{\mathrm{IMP}}$, and $b l a_{\mathrm{NDM}}$ ) were as previously described (Nordmann et al. 2011; Ellington et al. 2007). PCR products were separated in $2 \%(\mathrm{w} / \mathrm{v})$ agarose gels for 45 minutes at $100 \mathrm{~V}$ using tris-acetate EDTA buffer [40 mM Tris base, $5 \mathrm{mM}$ sodium acetate, $1 \mathrm{mM}$ EDTA, pH 8). Ethidium bromide (Promega) stained DNA products were visualised by ultraviolet (UV) transillumination, and images captured using an Alpha Imager ${ }^{\mathrm{TM}} 3400$ gel system (Alpha Innotech). Positive and negative controls, and a $100 \mathrm{bp}$ DNA ladder (Promega) were included in each gel run to determine approximate sizes of the PCR products. PCR products were prepared for sequencing using a Wizard SV gel PCR cleanup kit in accordance with the manufacturer's instructions. Purified DNA samples were sequenced at the Central Analytical Facility, University of Stellenbosch. Sequence identity was then confirmed using Chromas 2.4.3 and NCBI BLAST.

\section{Molecular typing of isolates by MLST}

MLST was performed only on bla $\mathrm{VIM}$ positive isolates as previously outlined by Curran et al. (2004). Chromosomal DNA was used as a template for amplification of the seven housekeeping genes $(\operatorname{acs} \mathrm{A}, \operatorname{aro\mathrm {E}}, \operatorname{gua\mathrm {A}}$, $m u t \mathrm{~L}, n u o \mathrm{D}$, pps $\mathrm{A}$, and $t r p \mathrm{E}$ ) and the resulting PCR products were analysed by agarose gel electrophoresis and purified as described above. Purified DNA samples were sequenced at the Central Analytical Facility, University of Stellenbosch. Based on the allele sequences each isolate was assigned a sequence type number using the allelic profiles available on the online P. aeruginosa MLST database (PubMLST, 2015). A phylogenetic tree was constructed through the MEGA 6 software using the neighbour joining (NJ) method with the Kimura 2 parameter model with gamma correction and 1,000 bootstrap replicates for all sequences. Analyses were performed using the concatemer sequence of the seven sequenced genes used for the MLST assessment.

\section{Results}

P. aeruginosa isolates $(\mathrm{n}=234)$ were recovered from urine, blood, pus, sputa and an environmental swab with the highest number of isolates being obtained from pus specimens. Seventy-nine isolates were resistant to carbapenems, with resistance to only imipenem $(\mathrm{n}=29)$, or both imipenem and meropenem $(\mathrm{n}=50)$. The bla $\mathrm{VIM}_{-2}$ negative carbapenem resistant $P$. aeruginosa isolates $(\mathrm{n}=66)$ were susceptible to most of the antimicrobial drugs tested when compared to the VIM positive isolates (refer to supplementary data). Multiple isolates were obtained from different specimens of the same patients. Fifteen bla $a_{\mathrm{VIM}-2}$ positive $P$. aeruginosa isolates were obtained from 9 different patients and one environmental sample in the haematology, oncology, and cardiac units. Limited clinical information was available.

All VIM-2 positive carbapenem resistant isolates (100\%) also showed resistance to ampicillin, amoxicillin, cefuroxime, cefuroxime axetil, cefoxitin, tigecycline, cefotaxime, nitrofurantoin, and trimethoprim with one isolate (R54) resistant to all drugs tested (Table 1).

All VIM-2 positive carbapenem resistant isolates showed susceptibility to colistin with one exception (R54). Other than colistin, a number of carbapenem resistant isolates showed susceptibility to amikacin (42\%), gentamicin (31\%), and ciprofloxacin (26\%) (Table 1). The MIC values for the VIM-2 positive isolates were 64 - $>256 \mu \mathrm{g} / \mathrm{mL}$ for imipenem and $32->256 \mu \mathrm{g} / \mathrm{mL}$ for meropenem.

MLST revealed five different novel sequence types, which are not listed on the PubMLST database. These sequence types were provisionally allocated, ST1, ST2, ST3, ST4 and ST5 while awaiting a response after submission of new sequences to the PubMLST database. The majority of isolates were the sequence type allocated ST1 (Table 2). 
Annear et al., Afr., J. Infect. Dis. (2017) 11 (2): 68-74

https://doi.org/10.21010/ajid.v11i2.9

Table 1: Clinical carbapenem resistant Pseudomonas aeruginosa isolates $(\mathrm{n}=15)$ identified as carrying the blaVIM-2 gene and their relevant clinical information and antibiotic susceptibility profiles

\begin{tabular}{|c|c|c|c|c|c|c|c|}
\hline Isolate No. & Specimen & Gender & Age & Resistant & Intermediate & Susceptible & $\begin{array}{l}\text { Health care } \\
\text { Facility }\end{array}$ \\
\hline R 8 & Pus & Female & 25 & $\begin{array}{l}\text { AMP, AMC, TZP, CXM, CXA, FOX, CTX, CAZ, FEP, IPM, MEM, } \\
\text { AMK, GEN, CIP, TGC, NIT, SXT }\end{array}$ & & CST & Hospital D \\
\hline R 22 & Pus & Female & 34 & $\begin{array}{l}\text { AMP, AMC, TZP, CXM, CXA, FOX, CTX, CAZ, FEP, IPM, MEM, } \\
\text { AMK, GEN, CIP, TGC, NIT, SXT }\end{array}$ & & CST & Hospital A \\
\hline $\mathrm{R} 27$ & Sputum & Male & 54 & $\begin{array}{l}\text { AMP, AMC, TZP, CXM, CXA, FOX, CTX, IPM, MEM, CIP, TGC, } \\
\text { NIT, SXT }\end{array}$ & CAZ, FEP & AMK, GEN, CST & Hospital B \\
\hline R 29 & Blood & Female & 50 & $\begin{array}{l}\text { AMP, AMC, TZP, CXM, CXA, FOX, CTX, CAZ, FEP, IPM, MEM, } \\
\text { AMK, GEN, CIP, TGC, NIT, SXT }\end{array}$ & & CST & Hospital A \\
\hline $\mathrm{R} 53^{\mathrm{a}}$ & Blood & Male & 32 & $\begin{array}{l}\text { AMP, AMC, TZP, CXM, CXA, FOX, CTX, CAZ, FEP, IPM, MEM, } \\
\text { AMK, GEN, CIP, TGC, NIT, SXT }\end{array}$ & & CST & Hospital A \\
\hline $\mathrm{R} 54^{\mathrm{a}}$ & Urine & Male & 32 & $\begin{array}{l}\text { AMP, AMC, TZP, CXM, CXA, FOX, CTX, CAZ, FEP, IPM, MEM, } \\
\text { AMK, GEN, CIP, TGC, NIT, CST, SXT }\end{array}$ & & & Hospital A \\
\hline $\mathrm{R} 55^{\mathrm{a}}$ & Urine & Male & 32 & $\begin{array}{l}\text { AMP, AMC, TZP, CXM, CXA, FOX, CTX, CAZ, FEP, IPM, MEM, } \\
\text { AMK, GEN, CIP, TGC, NIT, SXT }\end{array}$ & & CST & Hospital B \\
\hline R 56 & Pus & Female & 3 & AMP, AMC, CXM, CXA, FOX, CTX, IPM, MEM, TGC, NIT, SXT & $\mathrm{TZP}$ & $\begin{array}{l}\text { CAZ, FEP, AMK, } \\
\text { GEN, CIP, CST }\end{array}$ & Hospital C \\
\hline $\mathrm{R} 70^{\mathbf{b}}$ & Pus & Male & 57 & AMP, AMC, CXM, CXA, FOX, CTX, IPM, MEM, TGC, NIT, SXT & TZP & $\begin{array}{l}\text { CAZ, FEP, AMK, } \\
\text { GEN, CIP, CST }\end{array}$ & Hospital B \\
\hline $\mathrm{R} 71^{\mathrm{b}}$ & Pus & Male & 57 & AMP, AMC, CXM, CXA, FOX, CTX, IPM, MEM, TGC, NIT, SXT & TZP & $\begin{array}{l}\text { CAZ, FEP, AMK, } \\
\text { GEN, CIP, CST }\end{array}$ & Hospital B \\
\hline $\mathrm{R} 85^{\mathrm{c}}$ & Blood & Female & 49 & $\begin{array}{l}\text { AMP, AMC, TZP, CXM, CXA, FOX, CTX, CAZ, FEP, IPM, MEM, } \\
\text { AMK, GEN, CIP, TGC, NIT, SXT }\end{array}$ & & CST & Hospital A \\
\hline $\mathrm{R} 88^{\mathrm{c}}$ & Pus & Female & 49 & $\begin{array}{l}\text { AMP, AMC, TZP, CXM, CXA, FOX, CTX, CAZ, FEP, IPM, MEM, } \\
\text { AMK, GEN, CIP, TGC, NIT, SXT }\end{array}$ & & CST & Hospital A \\
\hline $\mathrm{R} 89^{\mathrm{c}}$ & Sputum & Female & 49 & $\begin{array}{l}\text { AMP, AMC, CXM, CXA, FOX, CTX, CAZ, IPM, MEM, TGC, NIT, } \\
\text { SXT }\end{array}$ & TZP & $\begin{array}{l}\text { CAZ, FEP, AMK, } \\
\text { GEN, CIP, CST }\end{array}$ & Hospital A \\
\hline $\mathrm{R} 90$ & Environment & N/A & N/A & $\begin{array}{l}\text { AMP, AMC, TZP, CXM, CXA, FOX, CTX, CAZ, FEP, IPM, MEM, } \\
\text { AMK, GEN, CIP, TGC, NIT, SXT }\end{array}$ & & CST & Hospital A \\
\hline R 91 & Pus & Male & 58 & AMP, AMC, CXM, CXA, FOX, CTX, IPM, MEM, TGC, NIT, SXT & TZP, CAZ, FEP & $\begin{array}{l}\text { AMK, GEN, CIP, } \\
\text { CST }\end{array}$ & Hospital B \\
\hline \multicolumn{8}{|c|}{$\begin{array}{l}\text { AMP-Ampicillin; AMC-Amoxicillin-clavulanic acid; TZP-Tazobactam; CXM-Cefuroxime; CXA-Cefuroxime Axetil; FOX-Cefoxitin; CTX-Cefotaxime; } \\
\text { CAZ-Ceftazidime; FEP- Cefepime; IPM-Imipinem; MEM-Meropenem; AMK-Amikacin; GEN-Gentamicin; CIP-Ciprofloxacin; TGC-Tigecycline; } \\
\text { NIT-Nitrofurantoin; CST-Colistin; SXT-Trimethoprim-sulfamethoxazole }\end{array}$} \\
\hline
\end{tabular}




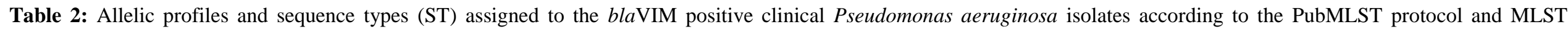
database and corresponding carbapenem MICs of each isolate. $(n=15)$

\begin{tabular}{|c|c|c|c|c|c|c|c|c|c|c|}
\hline \multirow{2}{*}{ Isolate } & \multirow{2}{*}{$\operatorname{acs} \mathbf{A}$} & \multirow{2}{*}{ aroE } & \multirow{2}{*}{ gua $\mathrm{A}$} & \multirow{2}{*}{ mut $\mathbf{L}$} & \multirow{2}{*}{ nouD } & \multirow{2}{*}{$\operatorname{pps} \mathbf{A}$} & \multirow{2}{*}{$\operatorname{trp} \mathbf{E}$} & \multirow{2}{*}{$* \mathbf{S T}$} & \multicolumn{2}{|c|}{$\mathrm{MIC}(\mu \mathrm{g} / \mathrm{ml})$} \\
\hline & & & & & & & & & Imipenem & Meropenem \\
\hline R 8 & 70 & 74 & 30 & 11 & 45 & 31 & 41 & 1 & $>256$ & 256 \\
\hline R 22 & 70 & 74 & 30 & 11 & 45 & 31 & 41 & 1 & 256 & 256 \\
\hline R 27 & 70 & 74 & 11 & 21 & 73 & 135 & 178 & 2 & 128 & 128 \\
\hline R 29 & 70 & 74 & 30 & 11 & 45 & 31 & 41 & 1 & $>256$ & $>256$ \\
\hline R 53 & 70 & 74 & 30 & 11 & 45 & 31 & 41 & 1 & $>256$ & $>256$ \\
\hline $\mathrm{R} 54^{\mathrm{a}}$ & 70 & 74 & 30 & 11 & 45 & 31 & 41 & 1 & $>256$ & $>256$ \\
\hline $\mathrm{R} 55^{\mathrm{a}}$ & 70 & 74 & 30 & 11 & 45 & 31 & 41 & 1 & $>256$ & $>256$ \\
\hline $\mathrm{R} 56^{\mathrm{a}}$ & 70 & 74 & 11 & 21 & 3 & 4 & 178 & 3 & 64 & 32 \\
\hline $\mathrm{R} 70^{\mathrm{b}}$ & 70 & 74 & 30 & 138 & 45 & 12 & 3 & 4 & 128 & 64 \\
\hline $\mathrm{R} 71^{\mathrm{b}}$ & 70 & 74 & 30 & 138 & 45 & 12 & 3 & 4 & 128 & 64 \\
\hline $\mathrm{R} 85^{\mathrm{c}}$ & 70 & 74 & 30 & 11 & 45 & 31 & 41 & 1 & $>256$ & $>256$ \\
\hline $\mathrm{R} 88^{\mathrm{c}}$ & 70 & 74 & 30 & 11 & 45 & 31 & 41 & 1 & $>256$ & $>256$ \\
\hline $\mathrm{R} 89^{\mathrm{c}}$ & 99 & 6 & 39 & 138 & 3 & 4 & 178 & 5 & 256 & 64 \\
\hline R 90 & 70 & 74 & 30 & 11 & 45 & 31 & 41 & 1 & $>256$ & $>256$ \\
\hline R 91 & 99 & 6 & 39 & 138 & 3 & 4 & 178 & 5 & $>256$ & 128 \\
\hline
\end{tabular}

a, $\mathbf{b}$ and c: refer to multiple isolates from the same patient, and includes different specimen type or different hospitals.

*Provisionally assigned sequence type ST while awaiting addition of new sequence types to PubMLST database. 
Multiple isolates from two patients were of the same sequence type while one patient had isolates of different sequence types. A dendrogram generated from the concatemer of the seven sequenced MLST genes of VIM-2 positive $P$. aeruginosa isolates $(\mathrm{n}=15)$ showed that with the exception of the isolate $\mathrm{R} 8$, the majority of the ST 1 isolates originated from hospital A and there was a confirmed environmental source (Figure 1).

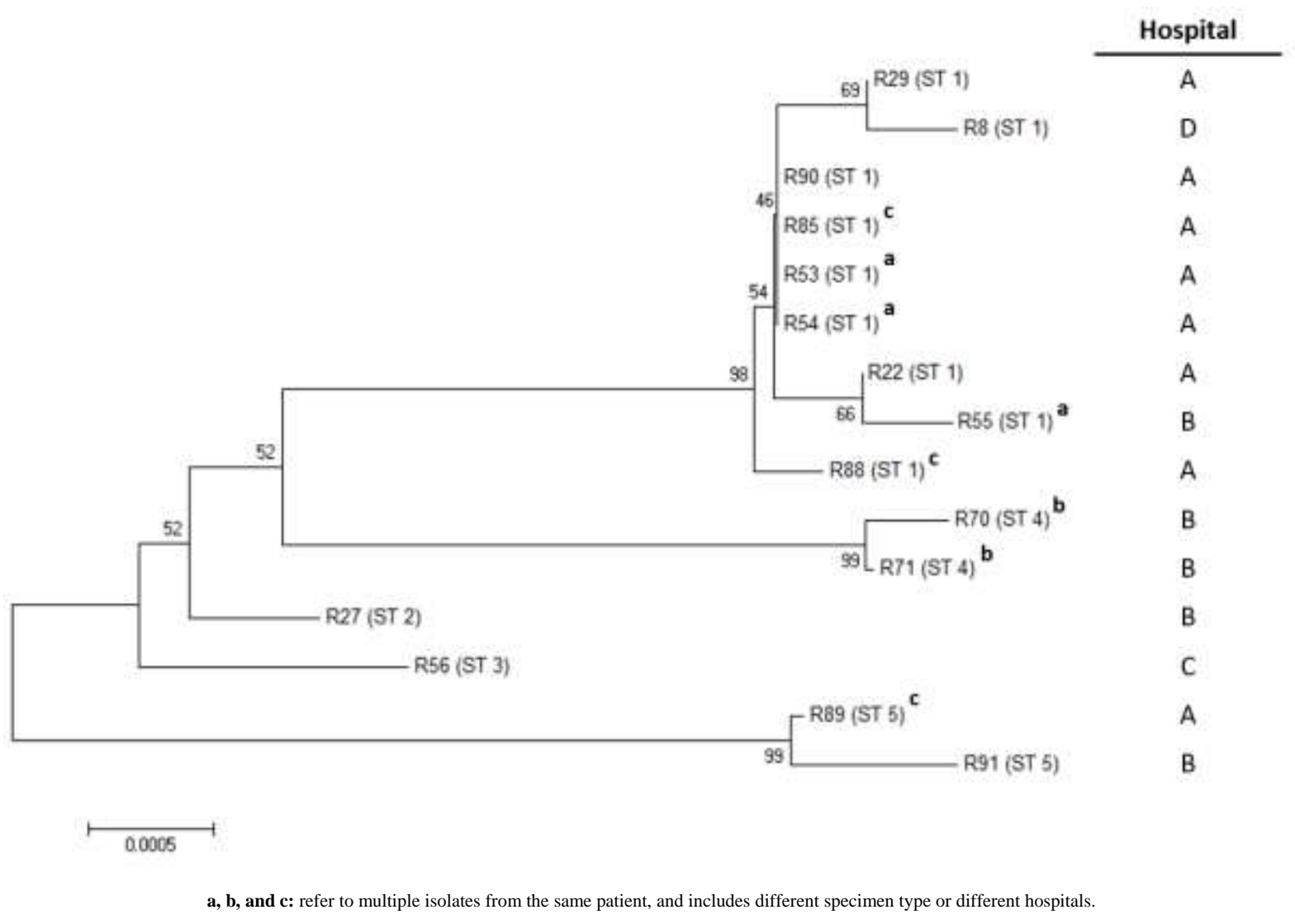

Figure 1. Neighbour-joining dendrogram generated from the concatemer of the seven sequenced MLST genes of fifteen blaVIM-2 positive $P$. aeruginosa isolates $(\mathrm{n}=15)$. The scale bar $(0.0005)$ indicates sequence divergence by the number of nucleotide substitutions per site. Bootstrap percentages retrieved in 1000 replications are displayed at the branch nodes.

\section{Discussion}

The emergence of multidrug resistant nosocomial bacteria has become a world-wide concern, especially with the emergence of resistance to last line antibiotics such as carbapenems. In this study, 35\% (81/234) of $P$. aeruginosa isolates exhibited resistance to carbapenems with $12 \%$ (29/234) resistant to only imipenem and 23\% (52/234) to both imipenem and meropenem. It is known that meropenem is more resistant than imipenem to enzymatic hydrolysis due to the 1- $\beta$-methyl group it contains within its structure (Shah and Isaacs, 2003). Similar findings were reported in Spain with carbapenem resistance in 39\% (175/448) of clinical P. aeruginosa isolates (Riera et al, 2011).

The MIC values of the blaVIM positive isolates were 64 - $>256 \mu \mathrm{g} / \mathrm{mL}$ for imipenem and $32->256 \mu \mathrm{g} / \mathrm{mL}$ for meropenem while MIC values were $\geq 8 \mu \mathrm{g} / \mathrm{mL}$ for blaVIM negative isolates. This exceeded the $8 \mu \mathrm{g} / \mathrm{mL}$ breakpoints recommended by both the EUCAST and CLSI guidelines for imipenem and meropenem resistance. MIC values $>32 \mathrm{ug} / \mathrm{ml}$ and $>256 \mu \mathrm{g} / \mathrm{mL}$ have been documented for imipenem and meropenem of carbapenemase producing $P$. aeruginosa by other researchers (Riera et al, 2011; Cho et al, 2015; Edelstein et al, 2013).

The metallo- $\beta$-lactamase VIM-2 encoding gene, bla $a_{\mathrm{VIM}-2}$, was found in 15 carbapenem resistant $P$. aeruginosa isolates. The production of carbapenemases is known to be one of the major mechanisms involved in carbapenem resistance (Rodríguez-Martínez et al, 2009). However it must be noted that only 15 of 81 carbapenem resistant $P$. aeruginosa contained the bla $a_{\mathrm{VIM}-2}$ gene. Carbapenem resistance in the bla $a_{\mathrm{VIM}-2}$ negative isolates is most likely due to other mechanisms such as active drug efflux pumps and porin gene mutations (Rodríguez-Martínez et al, 2009). It is also possible that other classes of carbapenemases rarely found in $P$. aeruginosa may have been present which were not screened for in this study. Cho et al, (2015) reported that of 61 carbapenem resistant $P$. aeruginosa isolates, obtained from a tertiary hospital in Daejeon, Korea, $25(41 \%)$ were metallo- $\beta$-lactamase producers. Cho et al, 
(2015) screened the isolates for 27 different $\beta$-lactamase encoding genes, but it was only VIM and IMP metallo- $\beta$ lactamases that were found within the $P$. aeruginosa isolates. Interestingly Cho et al (2015) reported that in each VIM and IMP positive isolate OXA-type carbapenemases were also present.

This study screened for the most common carbapenemases present in $P$. aeruginosa, hence future research could include detection of other carbapenemases. However, OXA-23 and OXA-51 carbapenemases have been reported in clinical P. aeruginosa isolates in Port Elizabeth (Gqunta, 2014). Riera et al, (2011) reported VIM-2, VIM-13 and VIM-20 as being the only metallo- $\beta$-lactamases found in the isolates screened at a hospital in Spain.

The MLST method of molecular typing for P. aeruginosa was used and it assesses the sequence of seven highly conserved housekeeping genes $(\operatorname{acs} \mathrm{A}, \operatorname{aro\mathrm {E}}, g a u \mathrm{~A}, m u t \mathrm{~L}, n u o \mathrm{D}, p p s \mathrm{~A}$, and $\operatorname{trp} \mathrm{E})$ and by comparing the sequence and combination of genes the clonal relationship between isolates can be assessed (Curran et al, 2004). It has been reported that isolates with the same sequence types (ST) can be considered as members of the same clone, and $P$. aeruginosa isolates that share at least five of the seven numbers within their allelic profile were regarded as members of the same clonal complex (PubMLST, 2015; Tiexeira and Merquior, 2013). Figure 1 illustrates that when phylogenetic analysis was applied using the concatemer of the seven sequenced MLST genes, isolates exhibiting the same sequence type all clustered together within the phylogenetic tree. This indicates a high likelihood of isolate relation and supports the sequence types applied to the $P$. aeruginosa isolates through the MLST protocol. It can also be seen that isolates of different sequence types differ greatly from each other within figure 1, which is expected.

Isolates R8, R22, R29, R43, R53, R54, R55, R85, R88, and R90 (unique sequence type allocated ST1) share identical allelic profiles and sequence types as determined by MLST. However, these are unique sequence types which have not been listed on the PubMLST database. Mudau et al (2013) conducted a study in Cape Town, South Africa, where the MLST protocol was utilized to type clinical $P$. aeruginosa isolates. When compared to the results of this study, a few similar allelic numbers were seen namely; mutL: 11, ppsA: 31 , and trpE: 41 . However there is diversity in the allelic profiles and sequence types of the isolates from Port Elizabeth and Cape Town studies (Mudau et al, 2013).

While the majority of the isolates were ST1 $(60 \%)$, these isolates were largely confined to the outbreak in Hospital A, spread via an environmental reservoir (R90), with only one other ST1 isolate (R8) being identified outside of hospital A. Furthermore, three P. aeruginosa isolates (R53, R55 and R56) from the same patient were of the same sequence type (ST1) and it should be noted that this patient was transferred from hospital A to hospital B. Another patient from hospital A was found to harbour two P. aeruginosa isolates (i.e R88 and R89) of different sequence types (ST1 and ST5 respectively). The source of the ST5 isolate in this patient is unknown.

Although it is acknowledged that there are limitations in the sample size and available clinical information, the diverse sequence types in the different hospitals highlights the occurrence of widespread carbapenem resistant $P$. aeruginosa isolates. Hospital B is the referral hospital and had four different sequence types, implying multiple introductions of the carbapenem resistant $P$. aeruginosa isolates which may be indicative of a more pervasive problem. The incidence of genes responsible for carbapenemase production in carbapenem-resistant Pseudomonas aeruginosa and genetic relatedness of these isolates in public healthcare facilities within the Port Elizabeth area is of concern and requires further investigation.

\section{Acknowledgements}

The authors would like to thank the Nelson Mandela Metropolitan University Research Themes grant for funding the study. Vanessa Pearce and the Bacteriology Laboratory staff at the National Health Laboratory Services, Port Elizabeth are acknowledged for their assistance with collection of cultures.

\section{References}

1. Baumgart, A.M., M.A. Molinari and A.C. Silveria. 2010. Prevalence of carbapenem resistant Pseudomonas aeruginosa and Acinetobacter baumannii in high complexity hospital. Brazil J Infect Dis. 14(5): 433-436.

2. Belén, A., I. Pavón and M.C.J. Maiden. 2009. Multilocus Sequence Typing. Methods Mol Biol. 551(1):129-140.

3. Cho, H., G. Kwon, S. Kim, and S. Koo. 2015. Distribution of Pseudomonas-Derived Cephalosporinase and Metallo- $\beta$ Lactamases in Carbapenem-Resistant Pseudomonas aeruginosa Isolates from Korea. J Microb Biotechnol. 25(7):11541162 .

4. Clinical and Laboratory Standard Institute. 2015. Performance standards for antimicrobial susceptibility testing: 25th Informational Supplement. CLSI M100-S25. Clinical and Laboratory Standard Institute, Wayne, PA.

5. Curran, B., D. Jonas, H. Grundmann, T. Pitt and C.G. Dowson. 2004. Development of a Multilocus Sequence Typing Scheme for the Opportunistic Pathogen Pseudomonas aeruginosa. J Clin Microbiol. 42(12):5644-5649.

6. Driscoll, J.A., S.L. Brody and M.H. Kollef. 2007. The Epidemiology, Pathogenesis and Treatment of Pseudomonas aeruginosa Infections. Drugs. 67(3):351-368.

7. Edelstein, M., E. Skleenova, O. Shevchenko, J. D’souza, D. Tapalski, I. Azizov, M. Sukhorukova, R. Pavlukov, R. Kozlov, M. Toleman, and T. Walsh. 2013. Spread of extensively resistant VIM-2-positive ST235 Pseudomonas aeruginosa in Belarus, Kazakhstan, and Russia: a longitudinal epidemiological and clinical study. Lancet Infect Dis. 13(1):867-76. 
8. Ellington, M. J., J. Kistler, D. M. Livermore and N. Woodford. 2007. Multiplex PCR for rapid detection of genes encoding acquired metallo- $\beta$-lactamases. J Antimicrob Chemother. 59(1):321-322.

9. Gomila, M., M. del Carmen Gallegos, V. Fernández-Baca, A. Pareja, M. Pascual, P. Díaz-Antolín, E. García-Valdés and J Lalucat. 2013. Genetic diversity of clinical Pseudomonas aeruginosa isolates in a public hospital in Spain. BMC Microbiol. 13(1):138-137.

10. Govender, S., T. Masunda and J. Black. 2015. VIM-2 carbapenemase producing Pseudomonas aeruginosa in a patient from Port Elizabeth. SAMJ 105(5):328.

11. Gqunta, K. 2014. Prevalence and molecular epidemiology of extended-spectrum beta-lactamase-producing and carbapenem-resistant Enterobacteriaceae, Acinetobacter baumannii and Pseudomonas aeruginosa in Port Elizabeth. Masters dissertation, Faculty of Science, Nelson Mandela Metropolitan University.

12. Hirsch, E. and V. Tam. 2010. Detection and treatment options for Klebsiella pneumoniae carbapenemases (KPCs): an emerging cause of multidrug-resistant infection. J Antimicrob Chemother. 65:1119-1125.

13. Jacobson, K.R., N. Minenza, M. Nicol and C. Bamford. 2012. VIM-2 metallo-b-lactamase-producing Pseudomonas aeruginosa causing an outbreak in South Africa. J Antimicrob Chemother. 10(1):1-2.

14. Meletis, G., N. Vavatsi, M. Exindari, E. Protonotariou, E. Sianou, C. Haitoglou, D. Sofianou, S. Pournaras and E. Diza. 2014. Accumulation of carbapenem resistance mechanisms in VIM-2-producing Pseudomonas aeruginosa under selective pressure. Europ J Clin Microbiol Infect Dis. 33(1):253-258.

15. Mudau, M., R. Jacobson, N. Minenza , L. Kuonza, V. Morris, H. Engelbrecht, M. Nicol, and C. Bamford. 2013. Outbreak of Multi-Drug Resistant Pseudomonas aeruginosa Bloodstream Infection in the Haematology Unit of a South African Academic Hospital. PLOS One. 8(3):1-7.

16. Nordmann, P., T. Naas and L. Poirel. 2011. Global spread of Carbapenemase producing Enterobacteriaceae. J Emerg Infect Dis. 17(10):1791-1798.

17. PubMLST. 2015. Pseudomonas aeruginosa MLST Database. Available: http://pubmlst.org/paeruginosa/. Last accessed 10th Nov 2015.

18. Queenan, A.M., and K. Bush. 2007. Carbapenemases: The Versatile $\beta$-Lactamases. Clin Microbiol Reviews. 20(3):440-458.

19. Riera, E., G. Cabot, X. Mulet, M. García-Castillo, R. del Campo, C. Juan, R. Cantón, and A. Oliver. 2011. Pseudomonas aeruginosa carbapenem resistance mechanisms in Spain: impact on the activity of imipenem, meropenem and doripenem. J Antimicrob Chemother. 66(9):2022-2027.

20. Rodríguez-Martínez, J.M., L. Poirel, and P. Nordmann. 2009. Molecular Epidemiology and Mechanisms of Carbapenem Resistance in Pseudomonas aeruginosa. Antimicrob Agents Chemother. 53(11):4783-4788.

21. Shah, P. and R. Isaacs. 2003. Ertapenem, the first of a new group of carbapenems. J Antimicrob Chemother. 52(4):538542 .

22. Tiexeira, L., and V. Merquior. 2013. Molecular typing methods for the genus Pseudomonas. In: Molecular Typing in Bacterial Infections. New York: Springer Science and Business Media: pp. 421-426. 